\title{
InP/Si heterostructure for high-current hybrid
}

\section{triboelectric/photovoltaic generation}

Vladislav A. Sharov ${ }^{l, 2}$, Prokhor A. Alekseev ${ }^{*}$, , Bogdan R. Borodin ${ }^{I}$, Mikhail S. Dunaevskiy ${ }^{I}$, Rodion R. Reznik ${ }^{3}$, and George E. Cirlin ${ }^{2}$

${ }^{1}$ Ioffe Institute, Saint-Petersburg 194021, Russia

${ }^{2}$ Saint-Petersburg Academic University, Saint-Petersburg 194021, Russia

${ }^{3}$ ITMO University, Saint-Petersburg 197101, Russia

*E-mail: (P.A.A) npoxep@gmail.com 

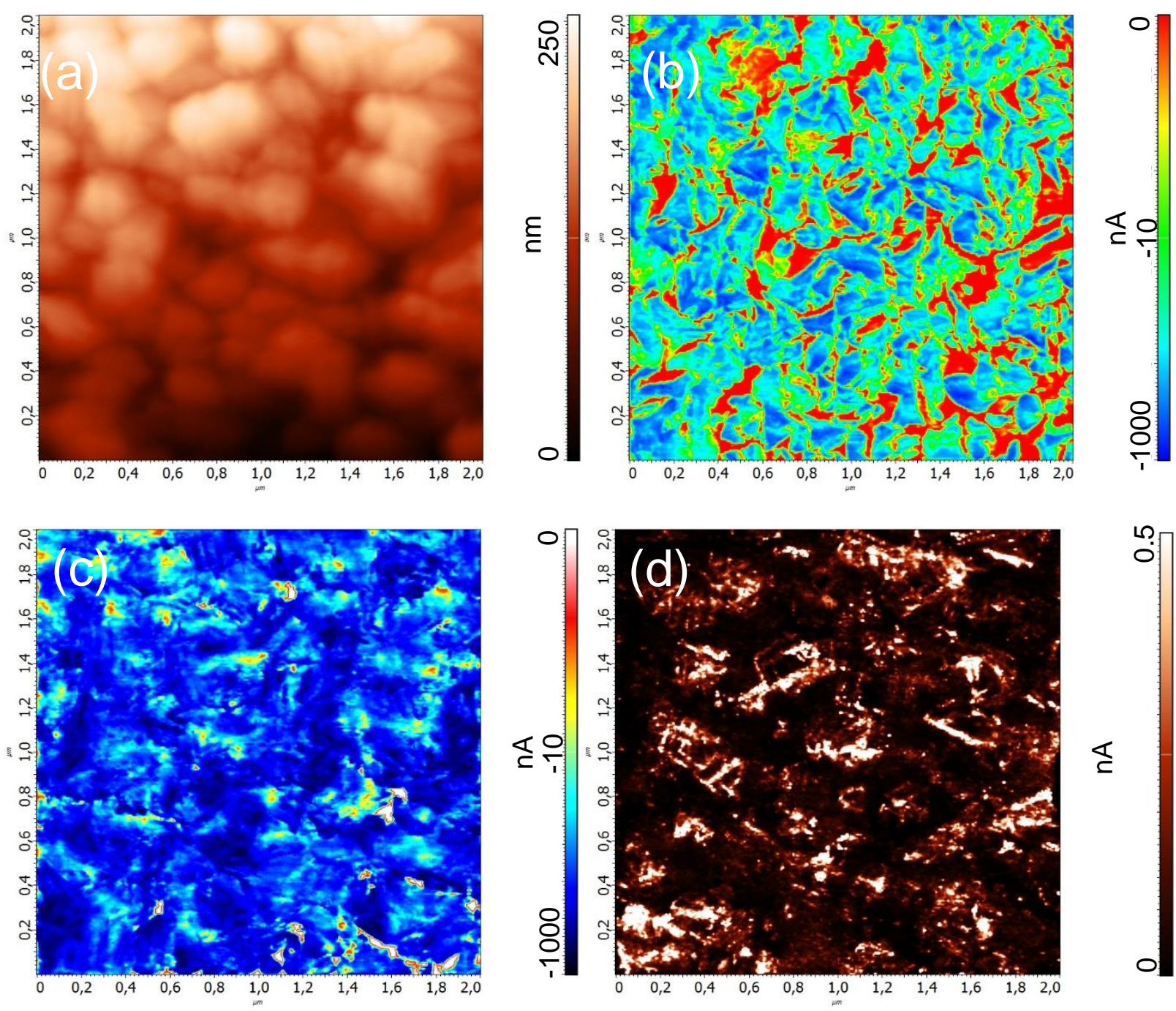

Figure S1. $2 \times 2$ um images of the same surface area obtained by DCP probe. (a) Topography image obtained in a constant force mode with pressure force $300 \mathrm{nN}$. (b) Photocurrent map obtained in a constant force mode with pressure force $300 \mathrm{nN}$. (c) Photocurrent map obtained in a constant height mode with pressure force $\sim 300 \mathrm{nN}$. (d) Dark current map (triboelectric current) obtained in a constant height mode with pressure force $\sim 300 \mathrm{nN}$. 

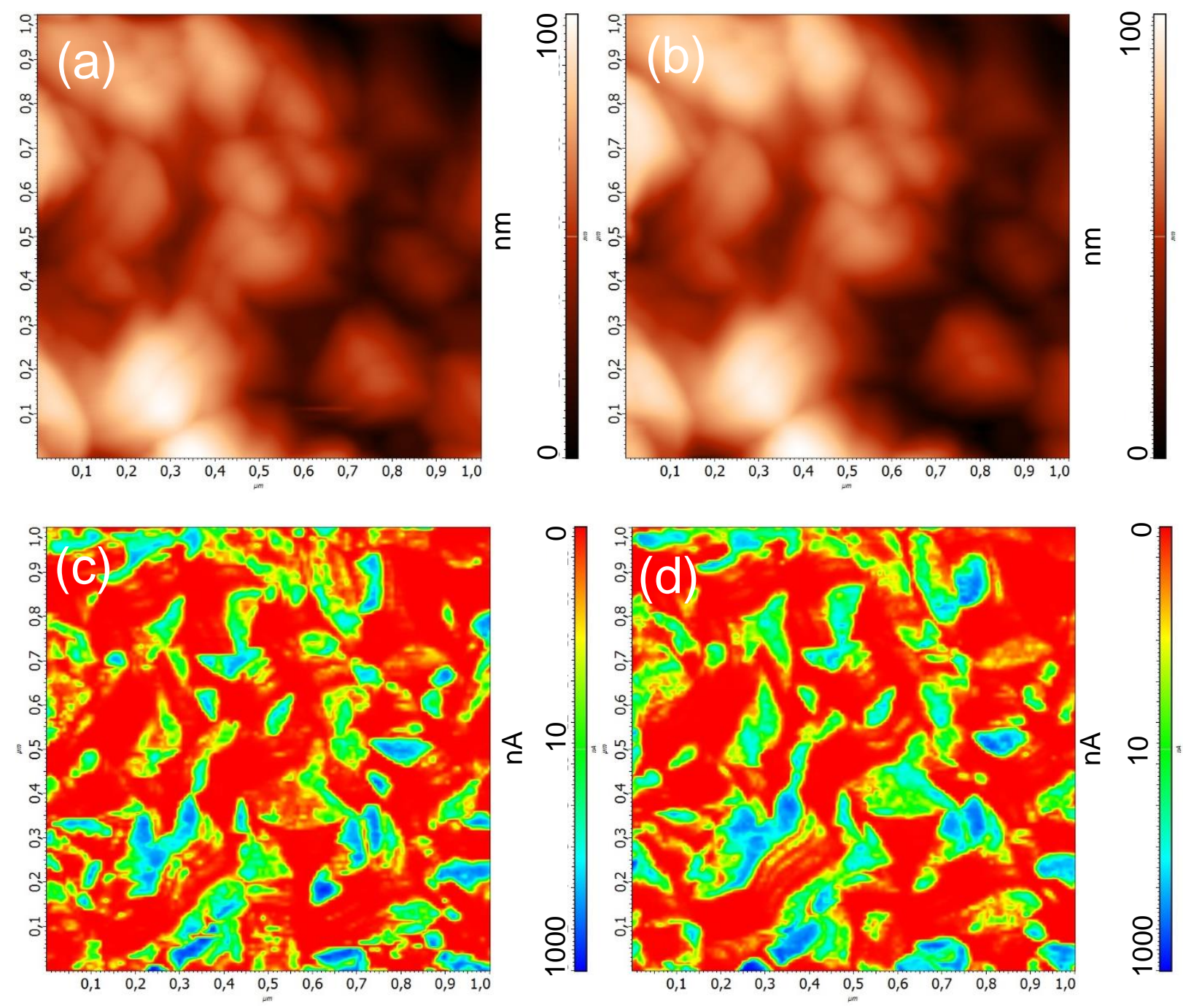

Figure S2. 1x1 um images of the same surface area obtained by DCP probe. (a-b) Topography images obtained in a constant force mode with pressure force $10 \mathrm{nN}$ for the first (a) and after 500th (b) scanning cycle. (c-d) Photocurrent maps obtained in a constant force mode with pressure force $10 \mathrm{nN}$ for the first (c) and after 500th (d) scanning cycle. 

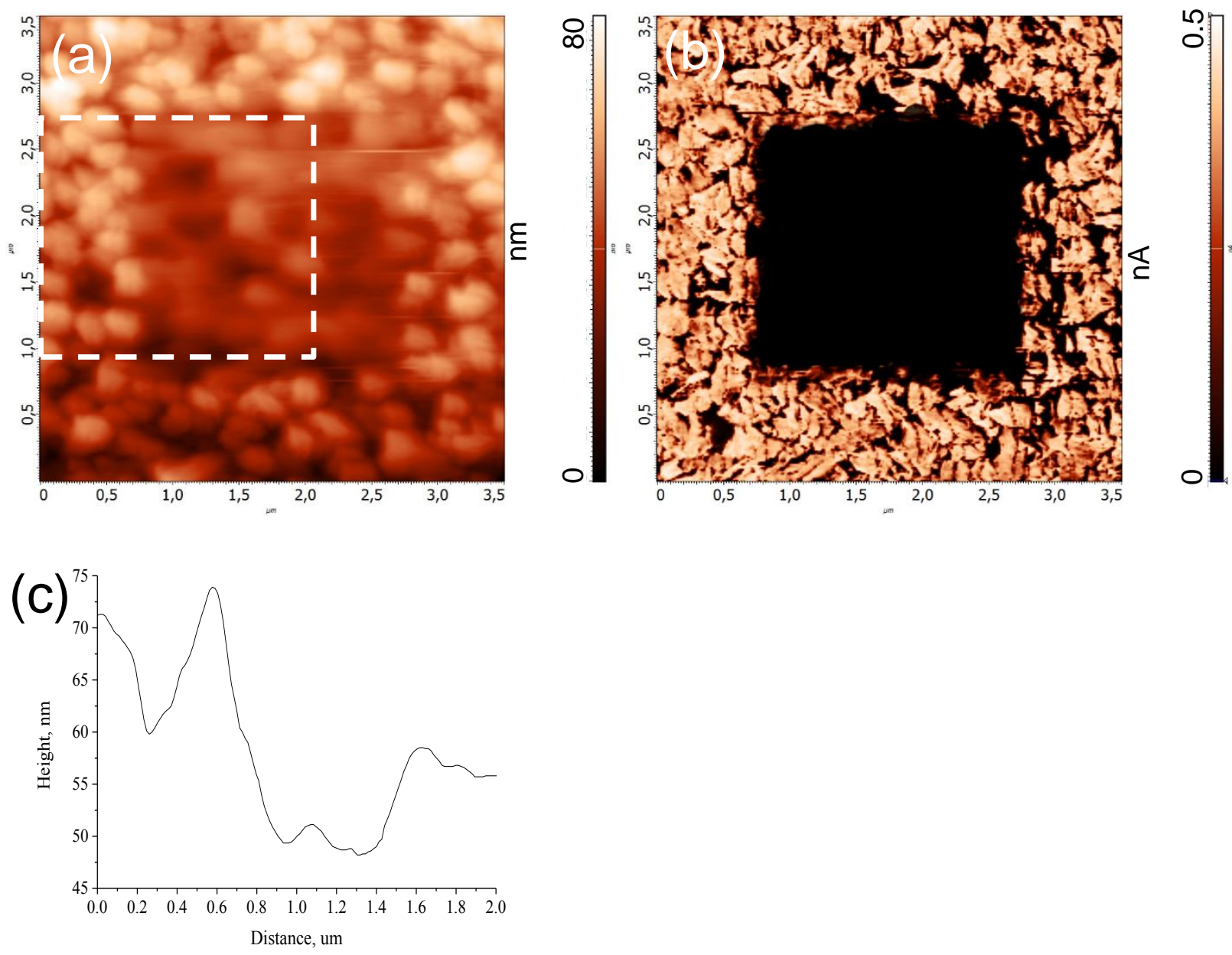

Figure S3. 3.5x3.5 um images obtained by DCP probe. Before the obtaining in the center of the scan a native oxide layer was removed by rubbing the surface $(2 \times 2 \mathrm{um})$ by the probe with the pressure force 1 uN. (a) Topography image obtained in a constant force mode with pressure force $300 \mathrm{nN}$ (b) Photocurrent map obtained in a constant force mode with pressure force $300 \mathrm{nN}$. (c) Averaged x-profile of the area marked by dashed line in Fig. S3a. 